\title{
Efficient Algorithm for the Detection of a Brain Tumor from an MRI Images
}

\author{
Ammar A. Radhi
}

\begin{abstract}
Medical image processing is considered as a very promising field due to its role in medical diagnoses of fatal diseases like cancers, with the availability of the advanced technology, detection of tumours has become easier nowadays. X-ray images and MRI images are examples which help in the earlier detection of different kind of tumours. However, further enhancement of these methods is currently undertaken. In this paper an algorithm for accurate detection of brain tumours is proposed, and based on the result obtained, it gives more accurate detection of a brain tumour in comparison to other methods like K-cluster, watershed algorithm, and threshold selection method. The algorithm is based on the application of a specific formula that segments the image very efficiently and isolates the tumour from the skull and other brain tissues based on the solidity and area. A comparison of the result of this algorithm with the previously mentioned methods is also proposed.
\end{abstract}

\section{General Terms}

Pattern recognition and brain cancer detection

\section{Keywords}

Enhancement, Segmentation, Morphological Operation, dilation, filtering

\section{INTRODUCTION}

Image processing is a method which is used to enhance an image and to extract the most important features from it. It usually applies a certain set of signal processing methods to the image which is two dimensional signals. MRIs are considered to be a better choice to study due to its high efficiency rate in comparison to images from X-rays, so the input here is MRI images. The MRI image of a patient is taken and there are several important procedures to be applied in order to enhance the image and detect the brain tumour, these procedures include the conversion of the image to greyscale since the MRI images may contain RGB colour and these colours should be removed in order to improve the further processes [1]. Next step would be the noise removal, as the images from any source even the MRI images may contain noise, so it's important to extract any unwanted signal from the image. Median filter is used in this paper since the use of this filter will preserve the edges of the important features while removing the noise [1]. Then the histogram of the MRI image is stretched, the histogram stretching process is important in order to simplify and enhance the extraction process of the objects of interest, which are not clear in the image. The resultant image is converted to the binary format, and some area opening procedure is applied to extract unimportant regions that occupy fewer than 50 pixels from the image. Dilation, which is a morphological operation, is used in this work to add pixels to the boundary of the tumour (and for other objects) in the image, the number of pixels added to the image depends on the structuring element which is a shape used to probe or interact with the image [2]. The formula used in this paper is applied to label the contents of the MRI image which includes the tumour region. Finally, the tumour region will be extracted depending on special features including the solidity and area of the objects that result from the labelling operation.

\section{LITERATURE REVIWE}

Several algorithms have been proposed for the detection of brain tumours, for a comparison purpose some methods and results will be mentioned in this paper

\subsection{K-Clustering Method}

$\mathrm{K}$-Clustering method is based on grouping pixels where all the pixels sorted in one group have a similar relationship. This method can for example sort pixels in an image based on their intensities and then it's due to the user to select the region of interest. The authors in [3] proposed an algorithm for the clustering techniques, this method first identify the cluster centres, then the distance between each cluster centre and each pixel will be calculated using the distance function in equation (1),

$m=\left|x_{i}-c_{j}\right|$ Eq. 1

Where;

$x_{1}, \ldots, x_{N}:$ is the input data points (pixels in the given image).

$j=1, \ldots ., k$, and $c_{j}:$ the cluster centres.

Then the data points will be distributed among the cluster centres according to the relation:

$x \in c_{j}$ if $\left|x-c_{j}\right|<\left|x-c_{i}\right|$ Eq. 2

This algorithm will choose the nearest distance between a pixel and a cluster centre to sort each pixel to that cluster centre in which it belongs [3] [4].

\subsection{Watershed Segmentation Method}

Watershed method is an intensity based method. It divides a group of pixels based on their intensities. Pixels which are having the same intensities are grouped together in order to divide the image into regions and then extract the tumour [5]. The mechanism of this method is like flooding each neighbouring local minima with water from below gradually, where each local minima represents a different region in the image, the water level rises at a uniform rate. Each pixel below the water level is marked as flooded; after increasing the water level, the flooded region will grow in size and after a while the two local minima will be drown with water. The water level from the two catchments basin will merge. The algorithm then constructs a barrier of one pixel thickness to separate the two regions. This operation continues until the 
algorithm constructs the boundary of each region in the image [6].

\subsection{Threshold selection Method}

Various methods have been used for selecting an image's threshold, which are mainly based on proposing a threshold for the histogram, for an ideal image the histogram has a sharp valley between the object and the background which make it possible to choose the threshold at the bottom of the valley. However, for a real image, it's not easy to detect the bottom of the valley accurately especially when the valley is flat. One example of a robot threshold selection method is the Otsu's method. This method suppose that the image is divided into two classes $C_{0}$ which represents the objects and $C_{1}$ which represents the background, by the selection of a threshold k; $C_{0}$ represents the pixels with level $(1, . ., \mathrm{k})$ while $C_{1}$ represents the pixels with level $(\mathrm{k}+1, \ldots, \mathrm{L})$, where (L) represents the grey levels of the image, by calculating the mean and variance in each class, the between class variance and the total class variance of the two classes, we get the following formula [7]:

$\eta(k)=\sigma_{B}^{2}(k) / \sigma_{T}^{2}$

Where,

$\sigma_{B}^{2}$ : represents the between class variance.

$\sigma_{T}^{2}$ : represents the total variance of levels.

Then the optimal threshold $\mathrm{k}^{*}$ would be the threshold which maximize $\eta\left(k^{*}\right)$ which is consequently maximizing the within class variance $\sigma_{B}^{2}\left(k^{*}\right)$ [7].

$$
\sigma_{B}^{2}\left(k^{*}\right)=\max _{1 \leq k \leq L} \sigma_{B}^{2}(k)
$$

\section{THE PROPOSED ALGORITHM}

The MRI brain image will be subjected first to some preprocessing procedures in order to enhance this image. The first stage will be converting the image from RGB image to greyscale image, then the output image will be applied to a median filter to extract the noise from this image, the noise free image is then applied to a contrast stretching process, this process is used to expand the intensity range of an image, as a result, some objects will be more clear and easy to detect [8]. the output image will be converted to the binary format and the resultant image will be subjected to some area opening operation in order to remove all connected components that have fewer than 50 pixels, some unimportant details of the target image will be removed as a result of this process, the tumour region normally covers more than this number of pixels, so the tumour as an object in the image will be safe to extract in the next part of the algorithm. Dilation is then recursively applied in order to construct a one pixel boundary around the tumour in the image and according to the following formula [9]:

$X_{K}=X_{K-1} \oplus B$ Eq. 5

Where:

$k$ : is $1,2,3$...until $X_{K}=X_{K-1}$.
$X_{K}$ : is a matrix of the first non-zero component in the given image.

$B:$ is a $12 * 12$ square structure matrix.

Figure (1) shows an example of the dilation operation.

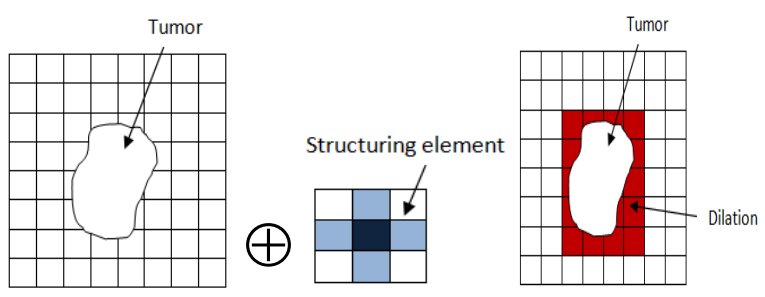

Fig.1. The dilation process of an MRI image

$X_{K}=\left(X_{K-1} \oplus B\right) \bigcap A$ Eq.6

Where:

A: is the original image.

After the dilation operation, the tumour will be isolated from the rest of the objects in the image and the next stage of the detection of the tumour will be comparing the resultant image with the original image according to the formula in equation(6) in order to extract the tumour region.

This algorithm is implemented using matlab with the following steps:

1. Detect a none zero component in the binary image.

2. Create a new matrix $(\mathrm{X})$ that contains only the none zero component detected from step 1 . The rest of the values in the matrix will be zeros

3. Perform the following recursive function $X_{K}=\left(X_{K-1} \oplus B\right) \cap A$ for $\quad \mathrm{k}=1,2,3, . . \quad$ until $X_{K}=X_{K-1}$.

4. The result in step 3 represents the first label.

5. Recreate the original image matrix $(\mathrm{A})$ to be $(\mathrm{C})$ by making each number one that belongs to the first label zero in order to detect the next label.

6. Detect the next none zero component from the image matrix created in 5 .

7. Repeat the function $X_{K}=\left(X_{K-1} \oplus B\right) \cap C$ if $X_{K}=X_{K-1}$ then result will represent the second label.

8. Repeat step 6 until no none zero component is detected.

The result from these steps is the segmentation of the image and labelling all the important objects within this image. However, not only the tumour will be labelled from this operation but some other objects, for instance, the skull and some of the brain tissues that are occupying more than 50 pixels, which remained even after the application of the area opening operation. Figure (2) shows the result after applying steps 1-6 on the MRI image. 


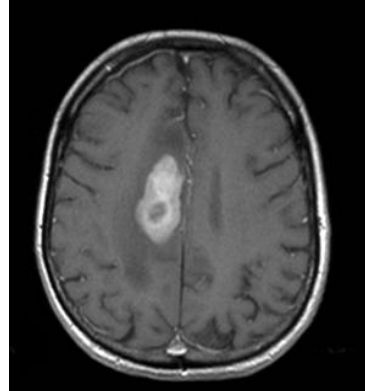

a

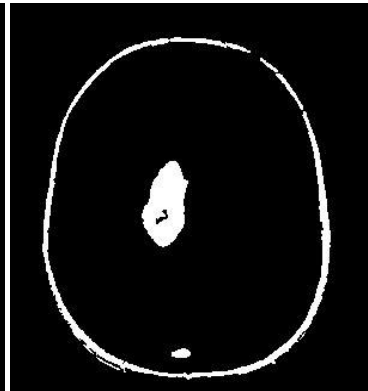

b
Fig 2 (a) The original image. (b) The result after applying equation (6).

The MRI image in figure (2) is labelled as follows: The skull is labelled as label 1 , the tumour is labelled as label 2 and some of the brain tissue is labelled as label 3. The following subsections discuss the process to extract the tumour alone

\subsection{Skull Stripping}

The task here is to extract the skull from the image; this process will make the labelling operation only applicable for the important tissues inside, including the tumour. To remove the skull from the image, the solidity of each label extracted from the application of equation (6) is calculated. The solidity determines the proportion of the pixels in the convex hull that are also contained in the region, where the convex hull specifies the smallest convex polygon that can contain the region. Thus the skull has the lowest value among other labels. Table.1 shows the solidity value of each label of the image in figure (2).

Table 1. Shows the solidity value for each label in the image of fig (2).

\begin{tabular}{|c|}
\hline Solidity \\
\hline 0.0652 \\
\hline 0.9156 \\
\hline 0.9423 \\
\hline
\end{tabular}

It can be shown from Table 1, that the first label, which represents the skull, has the lowest value. Thus the algorithm should be preceded by removing the lowest solidity value. The image in figure (3) represents an image before and after the removal of the skull.

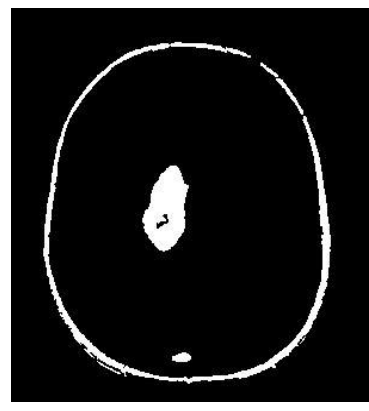

$\mathbf{a}$

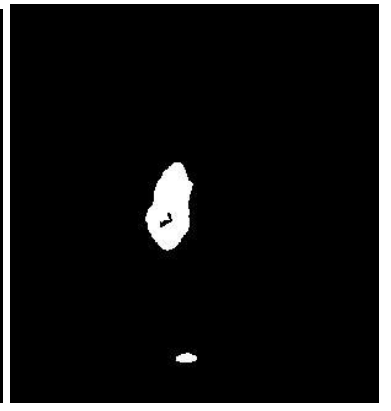

b
Fig.3. (a) The result of equation (6) before removing the skull. (b) The result after removing the skull.

\subsection{The Tumor Extraction}

The final step in this algorithm will be the extraction of the tumour from the image. This can be accomplished by the calculation of the area of each object (the area here is referred to the number of pixels occupied by the object) labelled by equation (6). Table 2, shows the solidity and the area of each label. From this table, the largest area is of label 1 which belongs to the skull, since the skull will be removed according to its low solidity, the remaining labels are 2 and 3 . Label 2 represents the tumour region and label 3 represents a part of the brain tissues. As it's tested, the second highest area always belongs to the tumour, thus by selecting the highest area while excluding the area of the skull; the tumour would have been detected. Figure (4) shows the result after the selection of the label that belongs to the tumour.

Table 2. Shows the solidity and area value for each label in the image of fig (2).

\begin{tabular}{|c|c|}
\hline Area & Solidity \\
\hline 3642 & 0.0652 \\
\hline 1780 & 0.9156 \\
\hline 98 & 0.9423 \\
\hline
\end{tabular}

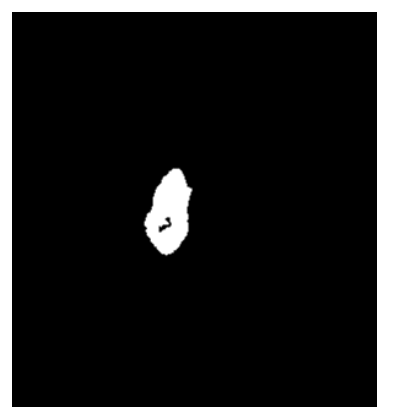

Fig.4. The extracted tumour.

The flow chart of the algorithm is shown in figure (5). 


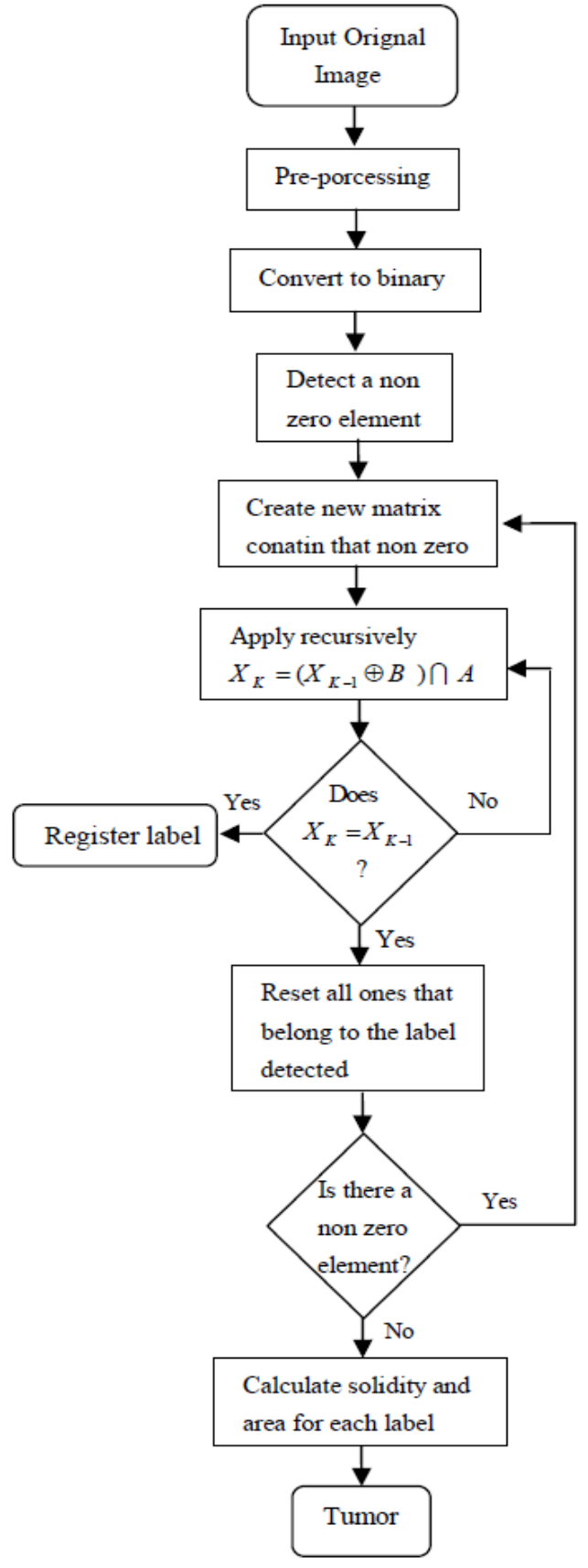

Fig (5). The flowchart of the algorithm

\subsection{Comparison to Other Tools}

As its stated before, the proposed algorithm produce a matrix that contains labels for the connected components in the binary image, however, in matlab there is a function known as 'bwlabeln' that can do the same work of this algorithm by labelling connected components, but it's not as efficient as the algorithm proposed, this algorithm is more efficient in labelling the component in the image and the number of labels produced can be easily managed by the structuring element, if the matrix of the structuring element is small more label will be produced and vice versa. The image shown in figure(2)(a) has only three labels if we apply the algorithm in this paper by using $24 * 24$ structuring matrix, whereas the same image will have 13 labels if we apply 'bwlabeln' matalb function. The number of labels effects the extraction of tumour from the image; in fact it will be so difficult to apply skill stripping and the tumour extraction procedure on any image to extract the area of interest. Table 3, shows the solidity, and area of each label of the image in figure (2) (a) if we apply 'bwlabeln' function on this image.

Table 3. Shows the area and solidity value for each label in the image of fig (2) in case of using bwlabeln function.

\begin{tabular}{|c|c|}
\hline Area & Solidity \\
\hline 3434 & 0.0618 \\
\hline 1 & 1 \\
\hline 1 & 1 \\
\hline 1 & 1 \\
\hline 31 & 0.7045 \\
\hline 1780 & 0.9156 \\
\hline 98 & 0.9423 \\
\hline 49 & 0.8305 \\
\hline 118 & 0.7284 \\
\hline 1 & 1 \\
\hline 1 & 1 \\
\hline 4 & 1 \\
\hline 1 & 1 \\
\hline
\end{tabular}

\section{THE RESULT OF THE ALGORITHM}

This algorithm has been tested for several patients' MRI images. All the images are in standard format that is Digital Imaging and Communication for Medicine (DICOM) [10], [11], [12], [13]. Figure (6) shows the application of this algorithm for the MRI image of the first patient

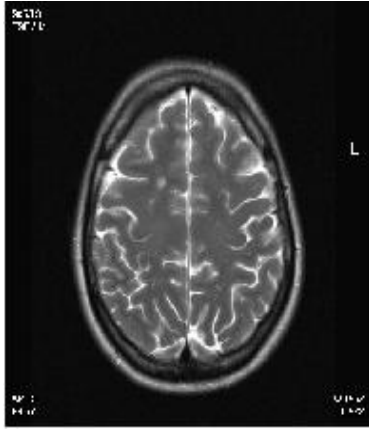

a

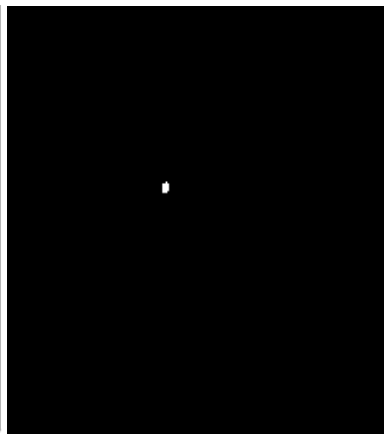

b
Fig. 6. (a) MRI image of patient no.1.(b) The extracted tumour.

As shown in figure (6), even for a small tumour the algorithm was able to distinguish it from the rest of the objects. The algorithm proposed showed a complete and accurate extraction of the tumour from the second MRI image of the second patient as shown in figure (7).

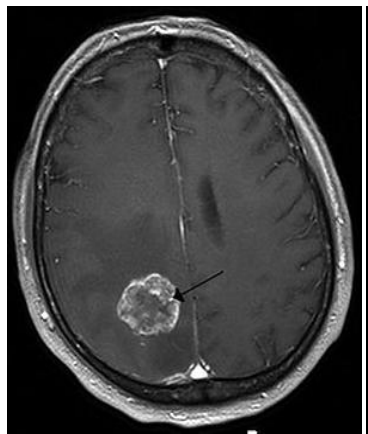

a

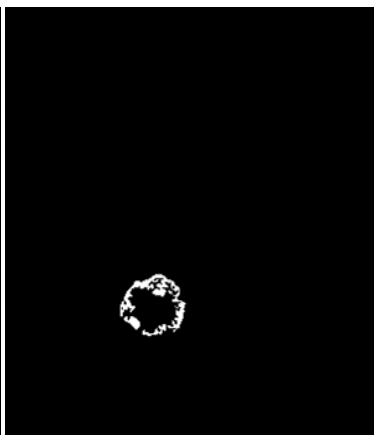

b
Fig.7. (a) MRI Image of Patient no.2. (b) The Extracted Tumour 


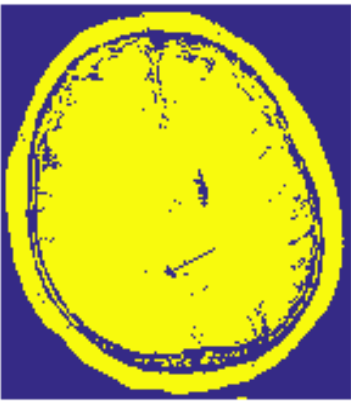

a

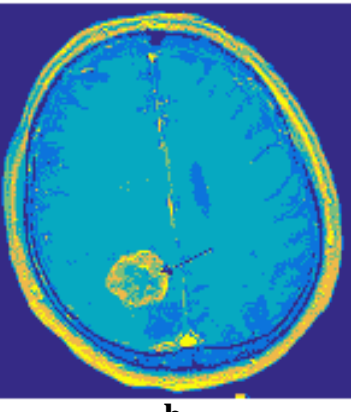

b
Fig.7. (a) Otsu's method with Two Classes Separation (b) Otsu's Method with Six Classes Separation

\subsection{Comparison of the Results}

A single sample of an MRI image is considered for a comparison purpose between the results obtained from this algorithm with the results obtained from the methods mentioned in section 2. If the MRI image of the second patient which is shown in figure (7) is considered, the algorithm proposed in this paper showed a complete detection of the tumour region as shown in figure (7) (b), while if we try to use k-clustering algorithm or watershed algorithm for the detection of the same tumour, then the result will be as shown in figure (8) (a) and figure (8) (b) respectively

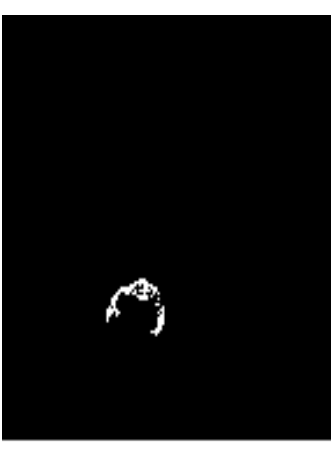

a

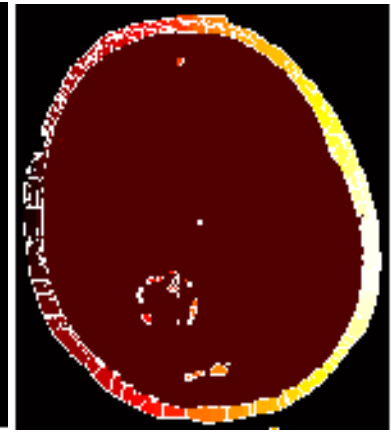

b
Fig 8. (a) Extracted tumour using K-clustering. (b) Extracted tumour using watershed algorithm of patient no.3

The both methods have failed to detect the tumour region completely. In case of k-clustering algorithm, although the skull was removed completely, the tumour was only extracted partially. Whereas in watershed algorithm, the skull has not been removed and the tumour region is not obvious. Now by applying the third method Otsu's method, which is an optimum threshold selection method, the results is as shown in figure (9) (b) and (c); in figure (9) (a) only two classes separation is used, while in figure(9)(b) six classes separation is used Even by increasing the separation classes to six, this method is still not able to detect the tumour region.

\section{CONCLUSION}

The aim of this work is to introduce an algorithm for the detection of brain tumours from an MRI image automatically, any accomplishment of such work will be of great help to doctors and patients and it will increase the healing chances in the early detection. The algorithm shows an accurate detection for several patients' cases studied in this paper. In comparison to other methods this algorithm extracts the tumour region completely and outlines the details of the tumour. In the future, this algorithm will be evaluated and developed to detect any other types of cancer with the help of some modifications that each type of cancer need. In addition, the algorithm could be developed further in order to automatically diagnose the cancer by analyzing the cells in any DNA Image. Accomplishing of such algorithm will replace the traditional methods of the diagnosing, which sometimes need an operation and could be painful for the patient.

\section{REFERENCES}

[1] Dr.K.Sakthivel, B.R.Swathi, S.VishnuPriyan, C.Yokesh: 'Analysis of Medical Image Processing and its Application in Healthcare'2016.

[2] Bernd Girod: 'Morphological Image Processing' 2013 Stanford University.

[3] Rohini Paul Joseph, C. Senthil Singh, M.Manikandan:'Brain Tumor MRI Image Segmentation and Detection in Image Processing' 2014.

[4] Nasir Ahmed, Kamisetty RamamohanRao: 'Feature Selection in Pattern Recognition', 'Orthogonal Transforms for Digital Signal Processing' SpringerVerlag, New York 1975

[5] Miss. Roopali R. Laddha, Dr.Siddharth A. Ladhake:'Brain Tumor Detection Using Morphological and Watershed Operator' 2014

[6] Dr.RashiAgarwal:'Watershed Algorithm for Segmentation' 2015.

[7] Nobuyuki Otsu: 'A threshold Selection Method from Gray Level Histograms’ 1979.

[8] Ahmad Dahlan, Prof.Soepomo Street, Janturan, Yogyakarta:'Image Enhancement Using Contrast Stretching on RGB and IHS Digital Image' 2007.

[9] 'Angel Johncy: Extraction of Connected Component without using BWLABEL in image processing'http://angeljohnsy.blogspot.com/2012/03/extr action-of-connected-components.html.accessedOctober 31,2014

[10] C.P. Loizou, V. Murray, M.S. Pattichis, I. Seimenis, M. Pantziaris, C.S. Pattichis: 'Multi-scale amplitude modulation-frequency modulation (AM-FM) texture analysis of multiple sclerosis in brain MRI images'. 2011.

[11] C.P. Loizou, E.C. Kyriacou, I. Seimenis, M. Pantziaris, S. Petroudi, M. Karaolis, C.S. Pattichis:'Brain white matter lesion classification in multiple sclerosis subjects for the prognosis of future disability' 2013.

[12] C.P. Loizou, M. Pantziaris, C.S. Pattichis, I. Seimenis:"Brain MRI Image normalization in texture analysis of multiple sclerosis'. 2013.

[13] C.P. Loizou, S. Petroudi, I. Seimenis, M. Pantziaris, C.S. Pattichis: 'Quantitative texture analysis of brain white matter lesions derived from T2-weighted MR images in MS patients with clinically isolated syndrome'. 2013. 